\title{
A New Technique for Precise Stellar Photometry: Application to Small-Scale Activity of EV Lacertae
}

\author{
B.E. Zhilyaev
}

Main Astronomical Observatory, National Academy of Sciences of Ukraine, Kiev 252650, Ukraine

Abstract: A new prefiltering technique (PFT) for precise stellar photometry is described. It has been proved that the prefiltered and added high-time resolution data provide an increase in $S / N$ ratio by factors $1.3-1.7$ in comparison with simple integration. The PFT allows to detect event amplitudes which are comparable to that caused by counting statistics. Adopting the PFT, our observations reveal small-scale flare events in the subsecond range in EV Lac.

\section{Brief sketch of the PFT algorithm}

For lack of space consider a specific application of the method. Let readings $x_{i}$ be mutually independent random variables, i.e. $\operatorname{cov}\left(x_{i}, x_{j}\right)=s^{2} \delta_{i j}$. Let us denote initial values of the mean count rate and the variance as $\bar{x}$ and $s^{2}$. Let the variation coefficient be $V_{0}=s / \bar{x}$. Then the $S / N$ ratio in the light curve is $S / N=1 / V_{0}$. Consider in addition raw readings $x_{i}$ in groups of three points, and readings $\tilde{x}_{i}$, smoothed by a moving-average three-point filter. Let us denote by $M$ and $D$ the expectation and the variance operators of the random variable. It is easy to show for raw and prefiltered data that

$$
y_{i}=x_{i-1}+x_{i}+x_{i+1}, M[y]=3 \bar{x}, D[y]=3 s^{2}, V_{3}=0.577 V_{0}
$$

and

$$
\tilde{y}_{i}=\tilde{x}_{i-1}+\tilde{x}_{i}+\tilde{x}_{i+1}, M[\tilde{y}]=3 \bar{x}, D[\tilde{y}]=19 / 9 s^{2}, \tilde{V}_{3}=0.484 V_{0} .
$$

Applying the PFT, the gain in $S / N$ is equal to 1.192 . As shown in detail by Zhilyaev et al. (1994), in the common case of an arbitrary $l$-point moving-average filter and an $m$-point addition, the gain is equal to

$$
\frac{V_{m}}{\tilde{V}_{l, m}}=\left(\frac{m}{\sum_{i=1}^{m} \sum_{j=1}^{m} r(i-j)}\right)^{1 / 2}
$$

with

$$
r(k)=\frac{l-k}{k}, \quad|k| \leq l-1,
$$

where $r(k)$ is the autocorrelation function of prefiltered data. The gain can reach a value of $1.3-1.7$ for appropriate values of $l$ and $m$. In this case, the radius of correlation of modified data is no more than $l-m+1$ points. 


\section{Application of the PFT to EV Lacertae}

High-time resolution observations were obtained with $0.01 \mathrm{~s}$ integration time in $B$-band with a high-speed photometer. The advantage of the PFT is shown in Fig. 1. The first panel shows a simple 5-point addition of readings, the second one is obtained by adoption of the PFT with $l=11, m=5$. The outburst seen in Fig. 1 is significant at the $>4 \sigma$ confidence level. Miscellaneous features of the flare-shaped event can be deduced from the PFT light curve. One can see a short-lived flare with an amplitude of $-0^{m} 4$, superimposed on an intensity drop of longer duration with an amplitude of $+2{ }^{\mathrm{m}} 2$. Significant intervals of the flare and of the drop are of the order of 1.5 and $3.5 \mathrm{~s}$, respectively. The paper attempts to describe the principle of operation of the prefiltering technique, and this example is a typical demonstration. Note that a reference star showed no presence of flare events at such a high confidence level during an overall observing run of half an hour.

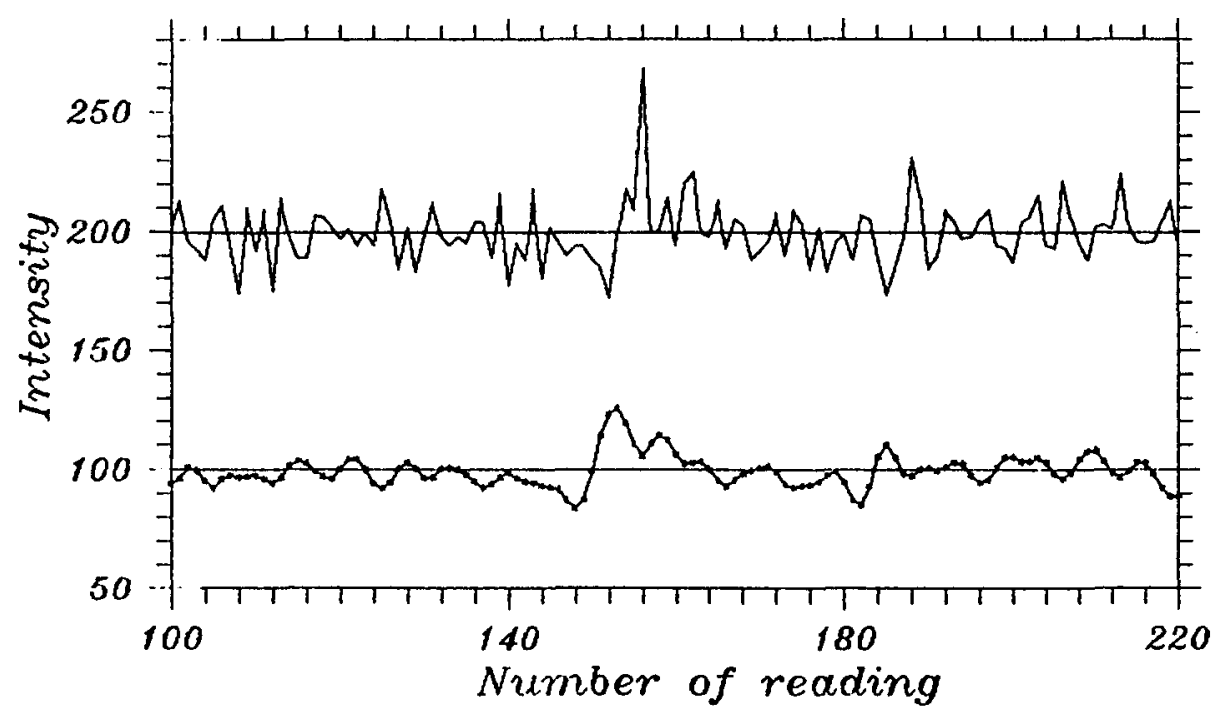

Fig. 1. EV Lac on 1992, Sept. 5, 22:05 UT, $B$-band. Time resolution is 0.05 s. Upper curve - raw data, lower curve - PFT light curve.

Acknowledgement: The research described in this publication was made possible in part by Grant No UCBOOO from the International Science Foundation.

\section{References}

Zhilyaev B.E., Romaniuk Ya.O., Svyatogorov O.A., 1994, Kinemat. Phys. Celestial Bodies 10, No. 6 (in press) 